\title{
The Effects of the Self-Talk Types and Task Complexity on the Accuracy of Forehand Topspin of Advanced Players
}

\author{
Mohammad Reza Sadeghian Shahi ${ }^{1}$, Rosa Rahavi Ezabadi ${ }^{2}$, , Najmeh Abootalebi ${ }^{1}$, \\ Parisa Moshiri ${ }^{1}$ \\ ${ }^{1}$ Department of Physical Education, Faculty of Humanities and Social Sciences, Yazd University, Yazd, Iran \\ ${ }^{2}$ Department of Motor Behaviour, Faculty of Sport Sciences, Alzahra University, Tehran, Iran

\section{Email address:} \\ Rsadeghian@yazd.ac.ir (M. R. S. Shahi), R.Rahvi@alzahra.ac.ir (R. R. Ezabadi), nabootalebi942@yahoo.com (N. Abootalebi), \\ moshiri_parisa@yahoo.com (P. Moshiri) \\ ${ }^{*}$ Corresponding author
}

\section{To cite this article:}

Mohammad Reza Sadeghian Shahi, Rosa Rahavi Ezabadi, Najmeh Abootalebi, Parisa Moshiri. The Effects of the Self-Talk Types and Task Complexity on the Accuracy of Forehand Topspin of Advanced Players. International Journal of Sports Science and Physical Education. Vol. 5, No. 2, 2020, pp. 16-20. doi: 10.11648/j.jisspe.20200502.12

Received: August 9, 2020; Accepted: August 26, 2020; Published: September 3, 2020

\begin{abstract}
The purpose of this study is to determine the effects of the motivational and instructional self-talk and task complexity on the accuracy of forehand top spin of table tennis in advanced players. The 30 male advanced players were divided into 3 groups ( 2 experimental and 1 control). The task complexity was determined by color of ball and place of table placement. In other words, sequence sending of the ball were changed after two balls and this trend continues. The keywords for motivational self-talk were "I can do" and " I correctly recognize", and for Instructional self-talk "pay attention" and "Close your paddle". Masters et. al test (2008) was used to measure the accuracy of forehand topspin. After the pre-test, subjects took part in 6 training sessions including 20 trails per session. After 48 hours, they participated in post-test. The data were analyzed by paired-samples t-test, one way ANOVA and Tukey post hoc test. Results showed that there is significant difference between the instructional and motivational self-talk in terms of task complexity. These findings suggested that instructional self-talk is the effective variable in performance of tasks that needs high complex decisions and accuracy.
\end{abstract}

Keywords: Instructional Self-Talk, Motivational Self-Talk, Task Complexity, Table Tennis

\section{Introduction}

In recent years, numerous intervention techniques have been used to develop and improve the performance and satisfaction of athletes in the area of applied psychology [1]. These techniques play an important role in improving performance [2]. In the meantime, cognitive strategies, by using effective patterns such as self-talk, goal setting, relaxation, and motivation regulation, have created positive changes [3]. A special type of these techniques is self-talk [1]. Huck Ford and Shvnkmzgr in defining, it refers to the internal and external dialogue uses by the performer, using a slow or loud voice, during performing the skills [4]. As Hardy stated, self-talk refers to oral expression (overt or covert) of athletes; essentially, self-talk has a multi-dimensional structure that one dimension is concerned with the performance [5].
Self-talk is a cognitive strategy and an educational tool that can be useful in learning and performing athletic sport skills. In addition to bringing a change in the thinking of the leaner and releasing him from a state of inactivity, it can lead to changing and modifying the current attitude with regard to the task being done [6]. Self-talk using the right keywords helps athletes to organize their thoughts, control, and focus on the parts of basic skills, or motivate themselves to try and enhance during their practice [7]. Theorists think of self-talk as a necessary component for mental programs related to training for skills, so many coaches take self-talk as a part of their plans [5]. In addition, Self-talk has positive and negative aspects, and two main functions: motivational and instructional. It seems that the motivational dimension of the self-talk, due to inspiration to further work and creating morale and confidence, can facilitate performance. On the other hand, the instructional dimension, due to easing the 
performance by focusing the appropriate attention, can improve technical information and tactics performance adopted [7]. Another technique that may affect motor performance and learning is the task complexity. Although the actual definition of the complexity of the task is not available, Wulf and Shea define a complex task as one in which a person cannot be so good during in a training session, with some degrees of freedom, possibly some ecological aspects [8].

The definition of task complexity only on the basis of certain characteristics or outcomes of the task is difficult. It is assumed that the complex task needs more reaction time [9]. It also needs more motor time, incorrect answers, and much instability [10] or more degrees of freedom [8]. Each of the features mentioned can affect the complexity of the task involved. On the other hand, the increase in the degrees of freedom cannot always lead to further complexity of the task. In fact, it may reduce the complexity of the task, like dexterity with the ball using one or both hands. While the degree of freedom is increased, the complexity is reduced [8]. Researchers working in the field of training variables (training pattern) and task complexity have concluded that the good training program should be based on factors such as the characteristics of the task; for example, difficulty [11].

The task complexity influences the initial levels and the amount of effective learning, usually, the results show that there may be some difference in the learning styles of simple and complex tasks [8]. The complexity of the task can have a powerful effect on both performance and response selection [12]; the increase or decrease in the amount of time between the decision on "what" and "how", by manipulating the speed of the ball or changing the size or weight of the ball, can have a dramatic effect on performance [13]; also, increasing the number of stimuli and responses can affect the quality of throw through creating ambiguity.

Rahavi et al showed that participants had better performance in the complex tasks, rather than the simple ones, showing the existence of a variable task complexity involved in learning [14].

According to the definitions mentioned earlier, it can be said that the task complexity could be due to two factors, one is associated with the task itself and the second one in connection with the training conditions, performance, or characteristics of the individual performer concerned. Guadagnoli \& Lee investigated task complexity in two different areas including task nominal complexity and task practical complexity. In fact, task nominal complexity is only related to the features of the task and has nothing to do with performance opportunities, training, or the skill level of the performer [15]. According to this definition, the task nominal complexity includes cognitive and motor factors required for the task.

Practical difficulty refers to the amount of challenge the task may impose on the individual, according to the skill level of the individual and the circumstances under which the task should be carried out. Another thing to bear in mind regarding the complexity of the task is the issue of expertise. Due to the expertise obtained, the processing ad memory requirements are automatically reduced. So if someone has already learned a task, it does not remain complex anymore. Under this condition, the reaction time and motor movement are reduced and the efficiency is increased [5]. One of the most important factors in information processing and the accuracy of the skill is decision-making. In fact, deciding on what to do in a given athletic position is based on the environmental information received by the participant, in relation to the knowledge base. So the difference in the perception and basic knowledge suggests that beginners are different from experts in making decisions.

More research carried out on decision-making in sports has been done on outdoor sports. In outdoor sports such as tennis and football, the environment is constantly changing. In dealing with the environmental demands, outdoor sports participants need to constantly make decisions that should be fast and accurate [16]. Based on the definitions discussed in this paper, the researchers aim to answer the question whether motivational and instructional self-talk with the task complexity, can influence the skill of topspin forehand among the advanced players? Also, the hypotheses of the present study is, there is a significant difference between motivational and instructional self-talk, with the task complexity on the accuracy of topspin forehand of advanced players.

\section{Methodology}

A quasi-experimental method (including two experimental groups and one control group) was employed in this field study. The design of the study was based on pre and post-tests with a control group. The sample, including 30 advanced players invited to an adult table tennis team who were in the first league and possed top positions in national and regional competitions before this research. They were in three homogenous groups, with each one consisting of 10 players with the age range of $28 \pm 1 / 6$.

\subsection{Measurement Tools}

To collect detailed data on age, physical activity, and physical health, the personal characteristics form was used.

$40-\mathrm{mm}$ balls (20 yellow and 20 white balls), table tennis ball thrower, Newgy Robo-pong 2000, digital video cameras Sony Alpha A6000, and standard table tennis table (Komann KBT-2012), were used in the present study.

\subsection{Data Analysis Method}

Research training protocol took two weeks and every week, included three sessions, and for each session a block were carried out (Masters et al., 2008). At first, the manner of the exercise protocol and the scoring for the strikes were orally explained to the participants and then this was followed by practical demonstration through the coach. To ensure a proper understanding of the protocol practiced by the participants, 
before the pre-test, the ability of individuals in choosing the correct answer was orally evaluated in twenty trials so that they were asked which side to place the ball. Subjects were then tested after training. To evaluate mean scores and compared to find any significant differences if necessary, to adjust the groups. Such differences were not observed among the groups. So, the participants were randomly divided into 3 homogenous groups regard to performance. Each group consisting of 10 players (motivational self-talk, instructional self-talk, and control). For each of the experimental groups, some information was provided about self-talk separately along with the keywords which were supposed to be used and explained. Then researchers asked them to use the keywords before doing the intended task. Then, in four-sessions (every alternate day), the protocol training acquired was carried out. During this exercise, self-talk intervention was applied just before the execution of hitting the topspin by the participants, in two groups (motivational self-talk and instructional self-talk).

The intervention took place in the two groups, such that the participants used words loud or in a whispering manner, which they repeated. The keywords used for motivational self-talk were "I can do" and "I correctly recognize" and for the instructional self-talk, saying" pay attention" and " adjust the angle (face) of the bat". After acquiring performance post-test was initiated on the participants.

The task consisted of topspin's strike of the balls to be sent at the end of the table. Depending on the direction of hitting and the area of the zone of table targeted, according to the color of the ball, the test stage and the turn of ball thrown were different. The way to create a curve and hit the forehand topspin was explained to the participants. At the end of the table, there was a ball thrower that could send the ball with a size of $40 \mathrm{~mm}$ at a rate of 15 balls per minute.

The balls were sent to the central line of the table, from a distance of $20 \mathrm{~cm}$ from the table. Forty balls (20 yellow balls and 20 white balls) were placed in the ball storage and were thrown randomly with different colors.

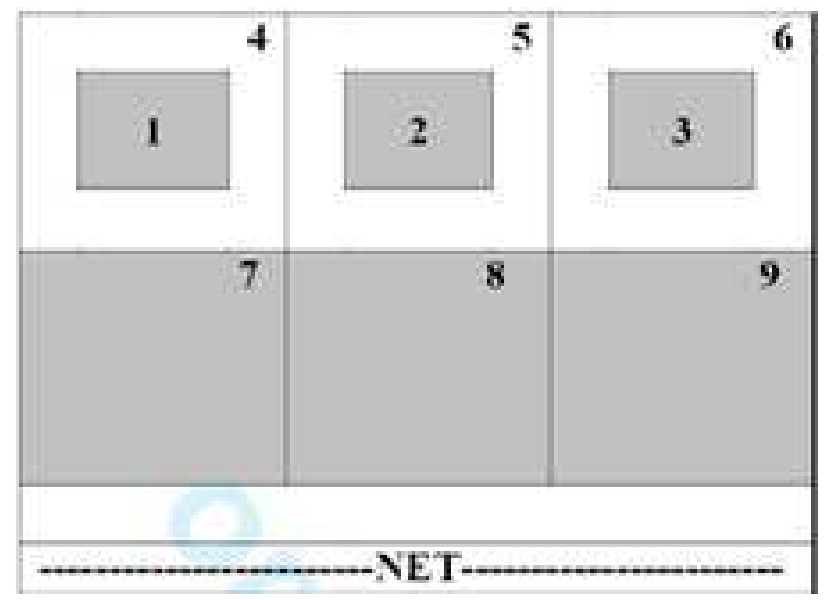

Figure 1. Table zoning.
The complexity of the task was determined by the color of the ball and where it was hit. In other words, after every two balls, the manner of hitting was changed, such that in the trials 1 and 2, white balls were hit towards the right and the yellow ones to the left; in the trials 3 and 4, the while balls were hit towards left and the yellow one towards the right and this trend continued. The maximum possible score was 60 points in the blocks of 20 trials [16].

\subsection{Statistical Method}

Descriptive statistics were used for drawing graphs, tables, and providing measures of central tendency. Shapiro Wilk normality test was used to investigate the normality of the data. The homogeneity of variance was tested by Levene's test. As for the analysis of data, to investigate the influence of the type of self-talk and task complexity on performance in the pre-test, the paired-samples t-test was used. On the other hand, one-way ANOVA was used in the post-test condition, and for the comparison of the groups, the Tukey post-hoc test was used. Data analysis was done using SPSS software, version 20, at 0.05 confidence level.

\section{Results}

Results obtained by one-way ANOVA showed no significant differences in the mean scores of three groups in the pre-test $\left(\mathrm{F}_{(2,29)}=0.734 ; \mathrm{P}=0.489\right)$, such that each of the groups was at a particular skill level; in fact, there was no difference in the skill level of the three groups before the intervention or treatment. Also, based on the scores obtained from the test groups, there was a statistically significant difference on $\mathrm{P}<0.05 \quad\left(\mathrm{~F}_{(2,29)}=10.161 ; \mathrm{P}=0.001\right)$. A comparison of the post hoc comparison using Tukey test showed that there was a significant difference in the post-test of instructional self-talk motivational self-talk group $(\mathrm{P}=0.003)$. In the instructional self-talk group, there was a significant difference with the control group $(\mathrm{P}=0.001)$. In the motivational self-talk group, there was no significant difference with the control group $(\mathrm{P}=1.000)$.

The paired-samples t-test was run to evaluate the effect of intervention on the scores of skilled players in the accuracy of topspin forehand in the post-test. Statistically, there was a significant increase in the scores related to the accuracy of forehand topspin group in the motivational self-talk group during the pre-test $(\mathrm{SD}=6.41, \mathrm{M}=33.1)$ until the post-test $(\mathrm{SD}=7.23, \mathrm{M}=44.8)\left(\mathrm{t}_{(9)}=1.055, \mathrm{P}=0.001\right)$. Also, there was no significant increase in the scores related to the accuracy of forehand topspin in the instructional self-talk group in the pre-test $(\mathrm{SD}=8.84, \mathrm{M}=29.5)$ until the post-test $(\mathrm{SD}=10.70$, $\mathrm{M}=30.9)\left(\mathrm{t}_{(9)}=8.718, \mathrm{P}=0.319\right)$. Furthermore, there was a no significant increase in the scores related to the accuracy of forehand topspin in the control group at the time of the pre-test $(\mathrm{SD}=5.42, \mathrm{M}=32.4)$ until the post-test $(\mathrm{SD}=6.65, \mathrm{M}=29.5)$ was $\left(\mathrm{t}_{(9)}=1.287, \mathrm{P}=0.230\right)$ (Table 1$)$. 
Table 1. The comparison of pre-test and post-test scores of different groups of the study.

\begin{tabular}{|c|c|c|c|c|c|}
\hline Groups & Time & Mean & Std. Deviation & t (two domains) & Sig. \\
\hline \multirow{2}{*}{ Motivational self-talk } & Pre-test & 33.1 & 6.41 & \multirow{2}{*}{1.055} & \multirow{2}{*}{0.001} \\
\hline & Posttest & 44.8 & 7.23 & & \\
\hline \multirow{2}{*}{ Instructional self-talk } & Pre-test & 29.5 & 8.84 & \multirow{2}{*}{8.718} & \multirow{2}{*}{0.319} \\
\hline & Posttest & 30.9 & 10.70 & & \\
\hline \multirow{2}{*}{ Control } & Pre-test & 32.4 & 5.42 & \multirow{2}{*}{1.287} & \multirow{2}{*}{0.230} \\
\hline & Posttest & 29.5 & 6.65 & & \\
\hline
\end{tabular}

\section{Discussion}

This study aimed to determine the effect of motivational and instructional self-talk and task complexity on topspin forehand accuracy in advanced players. The results showed that there was a difference on the effect of motivational self-talk and instructional self-talk and task complexity on the scores obtained upon hitting forehand topspin. The Tukey post hoc test was used to determine the difference between groups. Which showed significant difference between the instructional self-talk group and the control group. In other words, by employing instructional self-talk and task complexity to hit the forehand topspin, the accuracy was improved.

The results of the present study agree with those of finding Hardy et al; The comparison between instructional and motivational self-talk on skilled athletes in football shooting task, using the dominant and non-dominant foot. This indicated that instructional self- talk could be more effective in tasks which are based on accuracy. Also, the results of the present study support the findings. Investigated effect of instructional self-talk intervention on the performance of motor tasks, indicated positive effect on performing the task [17]. The results obtained by Zetou \& et al also showed that participants in the instructional self-talk group, in the final measurement, outperformed the control group [18].

But, based on the findings of Chang and colleagues having investigated the role of self-talk, featured on motor behavior and self-efficiency in the novice softball players. Motivational self-talk in the task to place the ball to a far distance, as compared to instructional and irrelevant self-talk, led to better performance. This showed disagreement with the current research [19]. One of the probable reasons for this inconsistency could be the age and expertise of the participants, as well as the nature of the task done. Thus, placing the ball at a far distance involves using more power which, in turn requires more motivation. The findings in the case of swimming task showed that the effect of motivational self-talk task on improving Swedish swimming was more than the instructional self-talk [20]. The reasons for the inconsistency of the results of this study can be attributed to the age of the participants and the nature of the task. In addition, the results of the current study opposes those obtained by Blakeslee \& Goff on improving the performance of the horse riders [21], and Harvey et al on the implementation of golf [22]. These two studies indicated that self-talk did not have positive effect on athletic performance. One of the reason for the difference between those research and the current study result, could be related to the gender and expertise level of the participants.

Self-talk can be regarded as a cognitive strategy and an instructional-psychological tool which can be used for practicing and learning athletic skills. In addition, it can lead to changing the thought process of the trainee. For instance it allows the player to overcome the inactivity state, thereby changing the thought patterns with regard to the task being done [6]. By employing self-talk and using the right keywords, athletes can develop their thoughts and there after control and focus on the major parts of the skill to motivate themselves to enhance attempts during exercise [7]. Planned Self-talk is can be an important variable in improving skills acquisition. With the improvement of skills, the nature of thoughts based on the level of professional achievement, the learning needs of the athlete can be varied in instructional and motivational self-talk. Self-talk by itself can motivate the thought process [23].

Based on the results obtained in this study, it is suggested that those coaches, who indulge in physical practice, employ self-talk as well as task complexity. All athletes in particular professionals doing complex task can use enhanced instructional self-talk diligently.

\section{Conclusion}

Decision - making is one of the most important components in information processing theory. On the other hand, Because of the differences in the perception and basic knowledge, experts differ from beginners in decisions making. So self-talk can help the decision-making process and the proper performance of skills by using behavior control rules that affect information processing functions.

\section{References}

[1] Miller, A. J. (2006). The influence of types and selection of mental preparation statements on collegiate cross-country runners' athletic performance and satisfaction levels. Miami University.

[2] Goudas, M. (2006). The effects of self-talk on throwing g-and j umping-events performance. Hellenic Journal of Psychology, 3 , 105-116.

[3] Papaioannou, A., Theodorakis, Y., Ballon, F., \& Auwelle, Y. V. (2004). Combined effect of goal setting and self-talk in performance of a soccer-shooting task .Perceptual and Motor Skills, 98 (1), 89-99. 
[4] Chroni, S., Perkos, S., \& Theodorakis, Y. (2007). Function and preferences of motivational and instructional self-talk for adolescent basketball players. Athletic Insight, 9 (1), 19-29.

[5] Hardy, J., Begley, K., \& Blanchfield, A. W. (2015). It's good but it's not right: instructional self-talk and skilled performance. Journal of Applied Sport Psychology, 27 (2), 132-139.

[6] Ziegler, S. G. (1987). Effects of stimulus cueing on the acquisition of groundstrokes by beginning tennis players. Journal of Applied Behavior Analysis, 20 (4), 405-411.

[7] Zinsser, N., Bunker, L., \& Williams, J. M. (2006). Cognitive techniques for building confidence and enhancing performance. Applied sport psychology: Personal growth to peak performance, 5, 349-381.

[8] Wulf, G., \& Shea, C. H. (2002) .Principles derived from the study of simple skills do not generalize to complex skill learning. Psychonomic bulletin \& review, 9 (2), 185-211.

[9] Klapp, S. T. (1995). Motor response programming during simple choice reaction time: The role of practice. Journal of Experimental Psychology: Human perception and performance, 21 (5), 1015-1027.

[10] Schmidt, R. A., Zelaznik, H., Hawkins, B., Frank, J. S., \& Quinn Jr, J. T. (1979). Motor-output variability: A theory for the accuracy of rapid motor acts. Psychological review, 86 (5), 415 .

[11] Keetch, K. M., \& Lee, T. D. (2007). The effect of self-regulated and experimenter-imposed practice schedules on motor learning for tasks of varying difficulty. Research quarterly for exercise and sport, 78 (5), 476-486.

[12] Fitts, P. M., \& Posner, M. I. (1967). Human Performance. Brooks. Cole, Belmont, CA (5), 7-16.

[13] Xiaopeng, Z. (1998). An experimental investigation into the influence of the speed and spin by balls of different diameters and weights. Science and racket sports II, 206-208.

[14] Rahavi, R., Aslankhani, M., Abdoli, B., \& vahabzadeh, A. (2009). The effects of scheduled practices (self-regulated and non-self-regulated) on learning of simple and complex tracking tasks. Development and Motor Learning (Harakat). (2), 65-85.

[15] Guadagnoli, M. A., \& Lee, T. D. (2004). Challenge point: a framework for conceptualizing the effects of various practice conditions in motor learning. Journal of motor behavior, 36 (2), 212-224.

[16] Masters, R., Poolton, J. M., Maxwell ,J. P., \& Raab, M. (2008). Implicit motor learning and complex decision making in time-constrained environments. Journal of motor behavior, 40 (1), 71-79.

[17] Zourbanos, N., Hatzigeorgiadis, A., Bardas, D., \& Theodorakis, Y. (2013). The effects of a self-talk intervention on elementary students' motor task performance. Early Child Development and Care, 183 (7), 924-930.

[18] Zetou, E., Vernadakis, N., Bebetsos, E., \& Makrari, E. (2012). The effect of self-talk in learning the volleyball service skill and self-efficacy improvement. Journal of Human Sport \& Exercise, 7 (4), 794-805.

[19] Chang, Y.-K., Ho, L.-A., Lu, F. J.-H., Ou, C.-C., Song, T.-F., \& Gill, D. L. (2014). Self-talk and softball performance: The role of self-talk nature, motor task characteristics, and self-efficacy in novice softball players. Psychology of Sport and Exercise, 15 (1), 139-145.

[20] Kolovelonis, A., Goudas, M., \& Dermitzaki, I. (2011). The effects of instructional and motivational self-talk on students' motor task performance in physical education. Psychology of Sport and Exercise, 12 (2), 153-158.

[21] Blakeslee, M. L., \& Goff, D. M. (2007). The effects of a mental skills training package on equestrians. The Sport Psychologist, 21 (3), 288-301.

[22] Harvey, D., Van Raalte, J., \& Brewer, B. (2002). Relationship between self-talk and golf performance. International Sports Journal, 6 (1), 84-91.

[23] Hatzigeorgiadis, A., Zourbanos, N., Mpoumpaki, S., \& Theodorakis, Y. (2009). Mechanisms underlying the self-talkperformance relationship: The effects of motivational self-talk on self-confidence and anxiety. Psychology of Sport and Exercise, 10 (1), 186-192. 